\title{
Diagnostic value of non-enhanced computed tomography in identifying location of ruptured cerebral aneurysm in patients with aneurysmal subarachnoid haemorrhage
}

\author{
Marcin Sawicki ${ }^{1}$, Krzysztof Kościukiewicz ${ }^{1}$, Dariusz Jeżewski ${ }^{2}$, Kornel Chełstowski ${ }^{3}$, Piotr Gołofit ${ }^{1}$, \\ Michał M. Skoczylas ${ }^{1}$, Michał Gębka ${ }^{1}$, Kornelia Grabizna ${ }^{4}$, Katarzyna Kołaczyk ${ }^{1}$, \\ Łukasz Zwarzany ${ }^{1}$, Leszek Sagan ${ }^{2}$, Wojciech Poncyljusz ${ }^{1}$ \\ ${ }^{1}$ Department of Diagnostic Imaging and Interventional Radiology, Pomeranian Medical University, Szczecin, Poland \\ ${ }^{2}$ Department of Neurosurgery, Pomeranian Medical University, Szczecin, Poland \\ ${ }^{3}$ Department of Laboratory Diagnostics, Pomeranian Medical University, Szczecin, Poland \\ ${ }^{4}$ Inter Medical Clinic, Kolobrzeg, Poland
}

\section{ABSTRACT}

Background. In patients with SAH and multiple aneurysms, the ruptured lesion must be identified to prevent recurrent bleeding.

Aim of the study. To assess the diagnostic value of non-enhanced computed tomography (NECT) in identifying the rupture site in patients with subarachnoid haemorrhage (SAH) and multiple aneurysms.

Material and methods. We included patients with SAH revealed by NECT and multiple aneurysms detected on computed tomography angiography (CTA) in whom a ruptured aneurysm was identified during neurosurgery. Two radiologists predicted the location of the ruptured aneurysm based on the distribution of the SAH and location of intracerebral haematoma (ICH) by NECT.

Results. Eighty-three patients with a mean age of $55.7 \pm 14.4$ years were included. Ruptured aneurysms were significantly larger (mean size $7.7 \pm 4.7 \mathrm{~mm}$ ) than unruptured aneurysms (mean size $5.9 \pm 4.5 \mathrm{~mm} ; \mathrm{p}=0.014$ ). Interobserver agreement was 0.86 $(\mathrm{p}<0.001)$. Overall sensitivity and specificity of radiological prediction were $78.3 \%(95 \% \mathrm{Cl}, 68.6 \%-87.1 \%)$ and $96.4 \%(95 \% \mathrm{Cl}, 94.3 \%$ 97.8\%) respectively. Overall PPV and NPV were $78.3 \%(95 \% \mathrm{Cl}, 67.6 \%-86.3 \%)$ and $96.8 \%(95 \% \mathrm{Cl}, 94.8 \%-98.1 \%)$ respectively. The sensitivity and PPV for aneurysms in the anterior communicating, anterior, and middle cerebral arteries appeared to be significantly higher than in other locations ( $p=0.015$ and 0.019 respectively). Analysis of independent predictive factors of correct radiological location revealed that $\mathrm{ICH}$ predisposes to a correct radiological diagnosis with an odds ratio of $8.57(95 \% \mathrm{Cl}, 1.07-68.99 ; p=0.03)$.

Conclusions. NECT has a high diagnostic value in identifying the source of bleeding in patients with multiple aneurysms for anterior circulation aneurysms, especially with coexisting ICH. For other locations, NECT is not reliable enough to base treatment decisions upon.

Key words: intracranial aneurysm, multidetector computed tomography, observer variation, subarachnoid haemorrhage (Neurol Neurochir Pol 2020; 54 (1): 47-53)

\section{Introduction}

Endovascular or neurosurgical treatment of patients after subarachnoid haemorrhage (SAH) with multiple aneurysms (up to $45 \%$ of all patients with intracranial aneurysms [1, 2]) is challenging. The correct identification of the ruptured aneurysm in cases of multiple aneurysms is of crucial importance, not only in endovascular but also in surgically treated patients.

Address for correspondence: Marcin Sawicki, Department of Diagnostic Imaging and Interventional Radiology, Pomeranian Medical University, Szczecin, Poland, e-mail: msaw@pum.edu.pl 
During open cranial surgery it is usually easy to identify the ruptured aneurysm which should be clipped first, before the remaining unruptured lesions.

But in cases where multiple aneurysms cannot be reached with one approach, correct localisation of the bleeding aneurysm is needed. Endovascular therapeutic options are being used increasingly because the results of randomised controlled trials indicate that endovascular coiling is associated with a better outcome $[3,4]$. However, endovascular therapy may be associated with greater uncertainty as to whether the bleeding aneurysm has been treated. In the presence of multiple aneurysms, errors about the location of the ruptured aneurysm can result in postoperative rebleeding. Therefore, correctly identifying every ruptured lesion is crucial for treatment planning.

Clinical signs can be helpful to locate the rupture site, but these are usually uncharacteristic and sometimes actually misleading. In the study by Nehls et al., clinical signs were indicative in only $7 \%$ of patients [5].

The accuracy of digital subtraction angiography (DSA) or magnetic resonance imaging (MRI) has also been assessed in several studies [6-9]. However, they showed poor reliability for DSA [6]. The accuracy of MRI has indicated that this technique is promising [7-9]. However, the application of MRI is limited due to insufficient availability, logistical challenges (including difficulty in scanning acutely ill patients), predisposition to motion artifacts, patient compliance, long study time, contraindications, and cost.

Routine diagnostic work-up of suspected SAH is usually based on non-enhanced computed tomography (NECT) and computed tomography angiography (CTA).

The sensitivity of CTA in detecting cerebral aneurysms is high, and known to exceed 95\%. However, in cases with multiple aneurysms CTA reveals all lesions with high sensitivity, but usually leaves the key question unanswered i.e. which of them has ruptured? Therefore, to increase the diagnostic value of CT in identifying the bleeding lesion, additional information is required. We hypothesised that NECT could provide just such additional information.

Several previous studies analysing different features in NECT as predictors of the rupture site have been equivocal and provided divergent results $[2,5,10,11]$.

Therefore, the aim of the present study was to assess the diagnostic value of NECT in identifying the location of a ruptured cerebral aneurysm in patients with SAH and multiple aneurysms.

\section{Materials and methods}

\section{Study participants}

For this retrospective cohort study, the approval of the local Bioethical Commission was obtained. Informed consent was waived. We reviewed the records of $256 \mathrm{pa}$ tients with aneurysmal SAH treated in the Department of Neurosurgery of our university hospital from March 2012 to November 2017. We included patients fulfilling the following criteria: NECT revealing SAH performed within 24 hours of the onset of symptoms; multiple aneurysms detected on CTA carried out immediately after the NECT; and ruptured aneurysms identified during neurosurgical treatment.

The exclusion criterion was poor quality NECT or CTA images not sufficient for reliable assessment.

\section{Non-enhanced CT protocol}

NECT was carried out using Somatom Sensation 64 and Somatom Definition AS+ scanners (Siemens, Erlangen, Germany). The scanning parameters were as follows: $120 \mathrm{kV}$, $380 \mathrm{mAs}$, range C1-C2 level to the vertex, FOV $220 \mathrm{~mm}$ and matrix $512 \times 512$, collimation of $64 \times 0.6 \mathrm{~mm}$ with a pitch of 1.2 , slice thickness $5 \mathrm{~mm}$, and slice increment $5 \mathrm{~mm}$ with Kernel H31s.

\section{Image interpretation}

NECT scans were assessed by a board-certified radiologist with 12 years of experience in neuroradiology ('Radiologist 1') and an uncertified radiology resident in the $4^{\text {th }}$ year of training ('Radiologist 2'). They were blinded to each other's findings, clinical data, the initial NECT report, the CTA results, and the findings of neurosurgery. The final decision concerning the predicted location of the ruptured aneurysm was reached by consensus.

As the aim of the study was limited to assessing solely the diagnostic value of NECT findings as predictors of aneurysmal rupture, NECT was analysed without knowledge of the distribution of multiple aneurysms in CTA. Information regarding the locations of particular aneurysms already detected by CTA would have biased our results.

Location and extent of SAH was classified according to the Hijdra scale that divides the subarachnoid space into 10 compartments [12]. Each compartment was given 0-3 points according to the amount of blood: $0=$ no blood; $1=$ small amount of blood; 2 = partially filled with blood; and 3 = completely filled with blood. This gave a minimum score of 1 and a maximum score of 30 .

Both radiologists predicted the site of the ruptured aneurysm based on the distribution of SAH and the location of the intracerebral haematoma ( $\mathrm{ICH})$ which was observed in some cases.

\section{Reference method}

Radiological prediction was verified by surgery as the reference standard. The site of craniectomy and the surgical approach was chosen after analysis of NECT and CTA findings by the neuroradiologist and neurosurgeon. CTA features which were regarded as associated with rupture included size, irregular shape, the presence of a nipple, focal spasm, or focal mass effect. 
During surgical treatment, the following findings were regarded as indications of an aneurysmal rupture: a healed thrombotic cap over the top of the aneurysm, a localised clot near the aneurysm, and very easy collapse (rupture) before or after clipping. The presence of at least one of these features was recognised as proof of aneurysmal rupture.

During the same surgical procedure, after clipping the ruptured aneurysm, all other accessible aneurysms were reached, confirmed as unruptured, and clipped if indicated.

\section{Data collection}

Demographic data including age and gender was noted. The predicted location of the ruptured aneurysm and Hijdra scale score were recorded by both radiologists. The location of the ruptured aneurysm identified during surgery was recorded. The size of the ruptured aneurysm was measured on CTA retrospectively analysed after surgical treatment. The size of an aneurysm was defined as its largest diameter. Both the two radiologists made three measurements of the aneurysm, and a mean value from these six measurements was used for further analyses.

Additionally, location and size of unruptured aneurysms based on CTA, presence of $\mathrm{ICH}$, and time from onset of symptoms to performing NECT, were noted.

\section{Statistical analysis}

Chi-square test was used to compare sensitivities, specificities, positive predictive values (PPVs) and negative predictive values (NPVs) of radiological diagnoses for different locations of aneurysms. U Mann-Whitney's test was applied to compare sizes of ruptured and unruptured aneurysms. Interobserver agreement was assessed with Kendall's tau-b value. A multivariate logistic regression model was used to find independent demographic and clinical predictors of false radiological diagnoses. $\mathrm{P}<0.05$ was considered statistically significant. For statistical analysis, STATISTICA 13.3 software (TIBCO Software Inc., Palo Alto, CA, USA) was used. A medical statistician evaluated the results of the analyses.

\section{Results}

Of the 256 patients whose records were reviewed, multiple aneurysms were detected in 83 (32\%) CTA examinations. None of the patients was excluded due to poor image quality. These 83 patients were included in the study. This group included $60(72 \%)$ females and $23(28 \%)$ males. The mean age of these patients was $55.7 \pm 14.4$ years (range 21-90). NECT was performed after a mean interval of $9.1 \pm 3.3$ hours following the onset of symptoms.

The distribution of ruptured aneurysms is presented in Table 1. The mean size of the ruptured aneurysm $(7.7 \pm 4.7 \mathrm{~mm}$, range 2-27) was significantly larger compared to the mean size of an unruptured aneurysm $(5.9 \pm 4.5 \mathrm{~mm}$, range $2-13$; $\mathrm{p}=0.014)$. ICH was detected in $25(30 \%)$ cases. The mean Hijdra score was $17 \pm 9$ (range 1-30).

Interobserver agreement between the two radiologists was very high; Kendall's tau-b value was $0.86(\mathrm{p}<0.001)$.

CTA revealed two aneurysms in $66(80 \%)$ patients, three in $16(19 \%)$ patients, and four in one (1\%) patient.

Based on NECT, the radiologists predicted the location of the ruptured aneurysm correctly (i.e. in line with surgical findings) in 65 of the 83 cases; hence, the sensitivity was $78.3 \%$ (95\% CI, $68.6 \%$ to $87.1 \%$ ) (Fig. 1 and 2). Detailed results of sensitivity, specificity, PPV and NPV are presented in Table 2.

Notably, the sensitivity for aneurysms located in the anterior circulation (anterior cerebral artery [ACA], anterior communicating artery [ACom], and middle cerebral artery [MCA]) appeared to be significantly higher than in other locations, including posterior circulation aneurysms: $92.3 \%$ (95\% CI, $80.6 \%$ to $97.5 \%$ ) vs. $56.7 \%$ (95\% CI, $37.7 \%$ to $74.0 \%$ ) with $\mathrm{p}=0.015$ (Chi-square).

We found overall specificity of radiological prediction of 96.4\% (95\% CI, 94.3\%-97.8\%).

Overall PPV and NPV were 78.3\% (95\% CI, 67.6\%$86.3 \%)$ and $96.8 \%$ (95\% CI, 94.8\%-98.1\%) respectively. As with the sensitivity, PPV for aneurysms in the anterior circulation (ACA, ACom and MCA) appeared to be significantly higher than in other locations: $85.7 \%$ (95\% CI, 73.2\%-93.2\%) vs. $62.9 \%$ (95\% CI, 42.5\%-79.9\%) with $\mathrm{p}=0.019$ (Chi-square).

We did not find significant differences in age, gender, Hijdra scale or size of the ruptured aneurysm between the groups with correct and incorrect radiological diagnoses. However, the prevalence of ICH was significantly higher in the group with a correct radiological diagnosis than in the group with an incorrect diagnosis (Tab. 3).

Table 1. Distribution of ruptured aneurysms

\begin{tabular}{lcc} 
& $\mathbf{n}=\mathbf{8 3}$ & Percentage \\
\hline ACA right & 1 & 1 \\
ACA left & 1 & 1 \\
ACom & 26 & 32 \\
MCA right & 13 & 16 \\
MCA left & 11 & 13 \\
ICA right & 8 & 10 \\
ICA left & 14 & 16 \\
BA & 4 & 5 \\
PCA right & 1 & 1 \\
PCA left & - & 0 \\
PICA right & 1 & 1 \\
PICA left & 3 & 4 \\
\hline $\begin{array}{l}\text { ACA - anterior cerebral artery; ACom - anterior communicating artery; MCA - middle cerebral } \\
\text { artery; ICA - internal carotid artery; BA - basilar artery; PCA - posterior cerebral artery; PICA - } \\
\text { posterior inferior cerebellar artery }\end{array}$ &
\end{tabular}



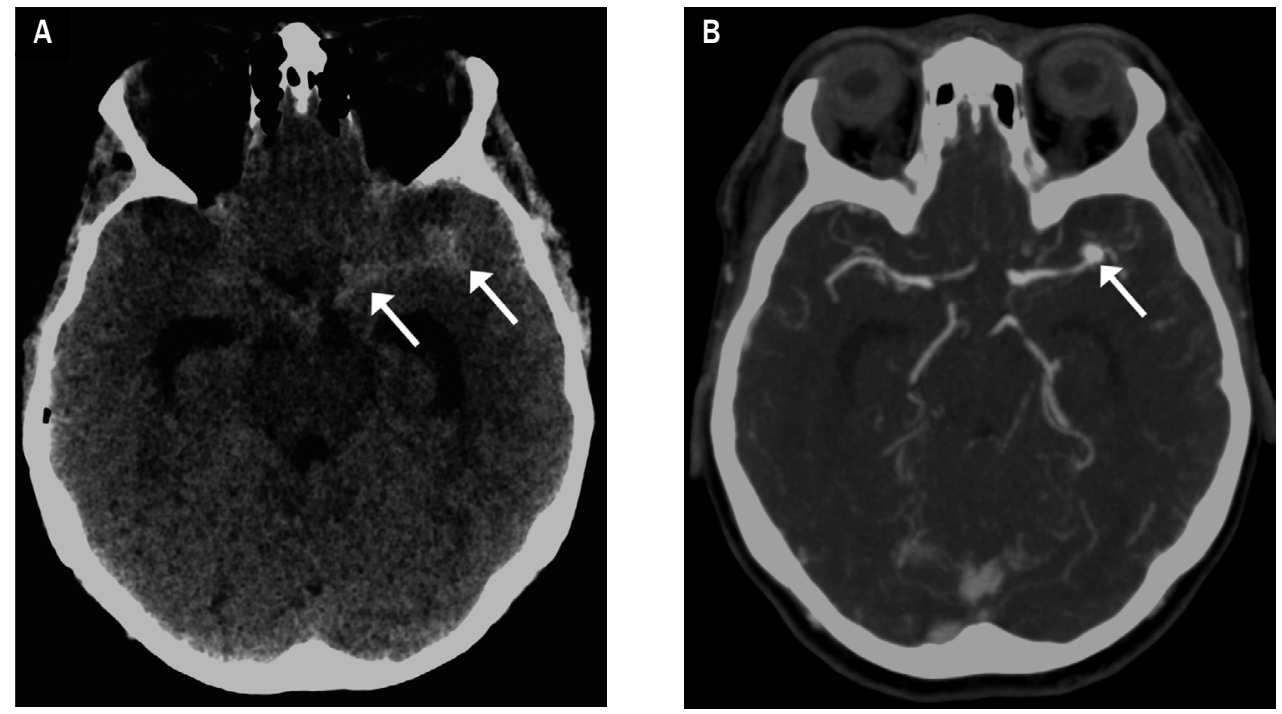

Figure 1. Patient with SAH and multiple cerebral aneurysms in whom localisation of ruptured aneurysm was correctly predicted by radiologists in NECT (A) on basis of clear lateralisation of SAH (arrows). CTA (B) reveals left MCA aneurysm (arrow)
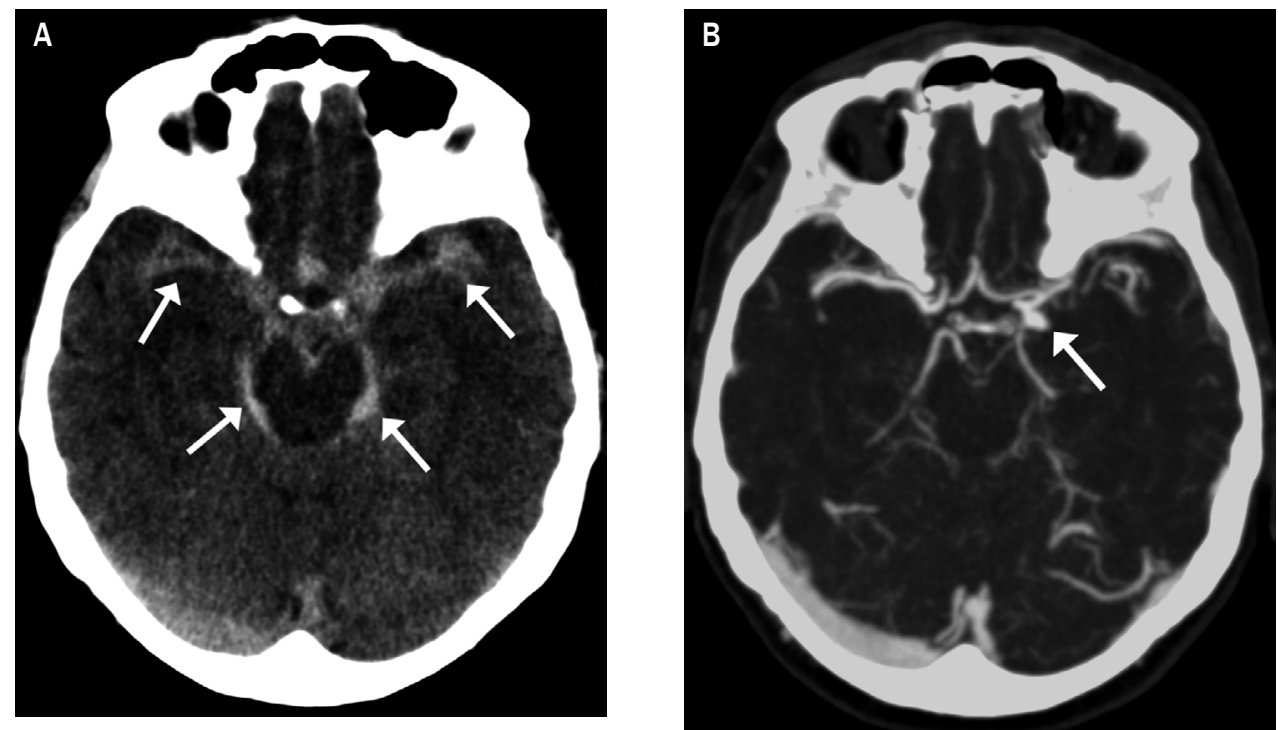

Figure 2. Patient with SAH and multiple cerebral aneurysms in whom localisation of ruptured aneurysm was incorrectly predicted by radiologists in NECT (A) showing bilateral, diffuse bleeding (arrows). Radiologists chose anterior communicating artery as localisation of ruptured aneurysm, while surgical verification revealed left internal carotid/posterior communicating artery aneurysm to be bleeding source. CTA (B) presents ruptured aneurysm (arrow)

Among 18 falsely predicted ruptured aneurysm locations, we found only one patient (with aneurysms located in the right MCA and the right internal carotid artery - ICA) in whom an unruptured aneurysm was revealed with CTA in a location wrongly considered by the radiologist to the location of a ruptured aneurysm. The radiologist selected an MCA aneurysm as the ruptured one, but surgical verification confirmed an ICA aneurysm as the source of bleeding. In the remaining 17 cases, although CTA detected multiple aneurysms, none of them was positioned in the location falsely predicted by the radiologists.
In seeking independent predictors of the correct location of the ruptured aneurysm by the radiologists, we analysed gender, age, Hijdra scale, size of the ruptured aneurysm, the presence of an $\mathrm{ICH}$, and the time from onset of symptoms to performing NECT. The presence of an ICH appeared to be an independent predictor of a correct radiological diagnosis, with an odds ratio of 8.57 (95\% CI, 1.07-68.99; $p=0.03$ ). Gender, age, Hijdra scale, size of the ruptured aneurysm, and the time from onset of symptoms to performing NECT were not independent predictors of correct results. 
Table 2. Results of radiological diagnosis for particular locations of ruptured aneurysms

\begin{tabular}{|c|c|c|c|c|}
\hline Location & Sensitivity & Specificity & PPV & NPV \\
\hline ACA & $50.0(2.6-97.3)$ & $100(94.4-100)$ & $100(5.5-100)$ & 98.8 (92.5-99.9) \\
\hline ACom & 92.3 (73.4-98.7) & $92.9(82.2-97.7)$ & $85.7(66.4-95.3)$ & 96.4 (86.4-99.4) \\
\hline MCA & 95.8 (76.9-99.8) & $93.2(82.7-97.8)$ & $85.2(65.4-95.2)$ & $98.3(89.4-99.9)$ \\
\hline ICA & $59.1(36.7-78.5)$ & $93.4(83.3-97.9)$ & $76.5(49.8-92.2)$ & 90.1 (80.1-95.6) \\
\hline $\mathrm{BA}$ & 75.0 (21.9-98.7) & 93.7 (85.2-97.7) & $37.5(10.2-74.1)$ & 98.7 (91.8-99.9) \\
\hline PCA & $0.0(0-94.5)$ & $100(94.4-100)$ & NA & 98.8 (92.6-99.9) \\
\hline PICA & $25.0(1.3-78.1)$ & 98.7 (92.2-99.9) & $50.0(2.7-97.3)$ & $96.3(88.8-99.0)$ \\
\hline Overall & $78.3(67.6-86.3)$ & 96.4 (94.3-97.8) & $78.3(67.6-86.3)$ & $96.8(94.8-98.1)$ \\
\hline
\end{tabular}

Results are presented as percentages and $95 \%$ confidence interval.

PPV — positive predictive value; NPV - negative predictive value; ACA — anterior cerebral artery; ACom — anterior communicating artery; $M C A$ - middle cerebral artery; ICA - internal carotid artery; BA — basilar artery; PCA — posterior cerebral artery; PICA — posterior inferior cerebellar artery; NA — not applicable

Table 3. Comparison of demographic and clinical data between groups with correct and incorrect radiological diagnoses. Prevalence of intracerebral haematoma $(\mathrm{ICH})$ significantly higher in group with correct radiological diagnosis

\begin{tabular}{|c|c|c|c|}
\hline Parameter & Correct radiological diagnosis $(n=65)$ & Incorrect radiological diagnosis $(n=18)$ & P value \\
\hline Age (years) & $54.8 \pm 15.2$ & $59.1 \pm 10.3$ & 0.2 \\
\hline Gender (f/m) & $44 / 21$ & $16 / 2$ & 0.2 \\
\hline Hijdra score (points) & $17.2 \pm 9.5$ & $18.1 \pm 9.1$ & 0.8 \\
\hline Ruptured aneurysm size (mm) & $8.0 \pm 4.9$ & $6.2 \pm 3.3$ & 0.17 \\
\hline $\mathrm{ICH}$ & $24(36.9 \%)$ & $1(5.6 \%)$ & 0.03 \\
\hline
\end{tabular}

\section{Discussion}

Our results indicate an overall sensitivity of $78.3 \%$ and specificity of $96.4 \%$ of NECT in correctly locating the ruptured aneurysm, with very high interobserver agreement.

Several previous studies assessing the amount and distribution of subarachnoid blood in NECT have shown divergent results. In their study of 168 patients, van der Jagt et al. found that only the location of a parenchymal haematoma predicted the site of rupture, but it was present in only $15 \%$ of cases [2]. They found that the distribution of blood was significant only for ACA and ACom aneurysms. Nehls et al. were able to locate the rupture site in $45 \%$ of 35 patients using NECT findings [5]. The sensitivity of NECT in identifying the ruptured lesion was about $70 \%$ in the study by Karttunen et al. of 180 aneurysms [10]. Latchaw et al. found that NECT could define the location of the ruptured aneurysm in approximately $80 \%$ of cases [11].

We found significantly higher sensitivity and PPV of the radiological diagnosis for ruptured aneurysms in the ACA, ACom and MCA compared to other locations, including the posterior circulation. A similar difference in sensitivity was also reported by van der Jagt et al. and Karttunen et al. $[2,10]$. We agree with these authors; this method seems unreliable for locating posterior circulation aneurysms.

We suggest that an uncharacteristic distribution of SAH from ruptured posterior circulation aneurysms could be caused by the complex anatomical structure of the subarachnoid space in this region. This may enable the blood jet to spread in many different directions. Takeuchi et al. described a case of a ruptured internal carotid/posterior communicating artery aneurysm in which blood clots were found in the cisterna magna, the fourth ventricle and the lateral ventricles [13]. Such blood distribution was misleading because it suggested that the ruptured aneurysm was located in the posterior fossa. Possible explanations considered by the authors included the formation of adhesions in the subarachnoid cisterns due to an earlier minor leakage, the orientation of the aneurysm (posteroinferior direction), and early wash out of the blood clot. Lee et al. presented two cases of bilateral ICA aneurysms that produced a contralateral distribution of clots on NECT [14]. They explained these misleading findings as due to adhesion and obliteration of the subarachnoid cisterns from a previous haemorrhage, with deviation of the direction of haemorrhage.

In contrast, the distribution of $\mathrm{SAH}$ from $\mathrm{ACA}, \mathrm{ACom}$ and MCA aneurysms is far more characteristic. In these cases, blood is typically present in the suprasellar cistern, along the cerebral falx and in the Sylvian fissures. However, in ACA and ACom aneurysms both Sylvian fissures are filled equally, whereas in MCA aneurysms usually clear lateralisation is observed, with larger amounts of blood observed in the hemisphere ipsilateral to the ruptured aneurysm.

It is worth underlining that our study provides novel information because, unlike others, we performed detailed analysis of each particular false result. We compared the locations of 
unruptured aneurysms revealed by CTA with the sites falsely diagnosed by radiologists. It appeared that there was only one case of the 18 patients whose NECT led the radiologist to choose a location in which an unruptured aneurysm was detected with CTA. Therefore, the remaining 17 results should be regarded as inconclusive.

In other words, radiologists chose the wrong aneurysm as a source of SAH in only one case.

Directly proportional dependence between the aneurysmal size and the risk of rupture confirmed by our study has already been widely discussed in the literature, including RCTs. However, this issue is beyond the scope of the present study.

Our analysis of the relationship of demographic and clinical features to the sensitivity of radiological diagnosis found that the presence of ICH independently predisposes to the correct result. This association was previously concluded by van der Jagt et al. and Karttunen et al. [2, 10].

There have also been publications assessing the usefulness of other imaging methods to localise the ruptured aneurysm. Studies using DSA to identify a site of rupture show limited value. Although this technique can provide absolute proof of the rupture site, which is extravasation of contrast material (the so-called "smoking gun" sign), this is rarely observed. Other signs, such as irregular shape, presence of nipples, focal spasm, focal mass or change, have been described $[15,16]$. However, these studies report discrepant results regarding the diagnostic value of these features as signs of aneurysmal rupture. Beck et al. compared location, size and shape of ruptured and unruptured aneurysms using biplanar and 3D rotational DSA techniques, and found only a single predictor of rupture. This appeared to be the multilobar shape of a 5-9 mm aneurysm [6]

Recently, detecting aneurysmal wall contrast enhancement by vessel wall MRI has appeared to be a valuable technique to distinguish between a ruptured and an unruptured aneurysm [7-9]. Nagahata et al. showed this method to be $98.4 \%$ sensitive and $81.9 \%$ specific in 117 patients [7]. However, the application of MRI is limited due to insufficient availability, logistical problems (including difficulty in scanning acutely ill patients), predisposition to motion artifacts, patient compliance, long study time, contraindications, and cost.

NECT is not subject to the same limitations as MRI, and is performed as a standard procedure as a method of choice in patients with suspected SAH. Therefore, NECT could be useful in managing patients with multiple cerebral aneurysms.

\section{Study limitations}

The main limitation of our study is the moderate overall number of participants. This limitation applies especially to low numbers of aneurysms in the ACA and in posterior circulation, reflected by broad ranges of $95 \%$ confidence intervals in results for these locations. This could possibly bias the results.

Studies including a sufficient number of ACA and posterior circulation aneurysms are needed to reliably assess the accuracy of NECT in identifying these locations as a bleeding source.

\section{Conclusions}

In patients with SAH and multiple cerebral aneurysms, NECT can identify the source of bleeding with high accuracy for ACA, ACom and MCA aneurysms, especially with the presence of ICH. For other locations, the method seems to be not accurate enough to be relied upon to make treatment decisions. However, in combination with CTA, the ruptured aneurysm can be identified in almost every patient.

Declarations of interest: The authors declare no conflict of interest.

Funding: No funding source to declare.

\section{References}

1. Rinne J, Hernesniemi J, Puranen M, et al. Multiple intracranial aneurysms in a defined population: prospective angiographic and clinical study. Neurosurgery. 1994; 35(5): 803-808, doi: 10.1227/00006123199411000-00001, indexed in Pubmed: 7838326.

2. van der Jagt M, Hasan D, Bijvoet HW, et al. Validity of prediction of the site of ruptured intracranial aneurysms with CT. Neurology. 1999; 52(1): 34-39, doi: 10.1212/wnl.52.1.34, indexed in Pubmed: 9921845.

3. Lindgren A, Vergouwen MDI, van der Schaaf I, et al. Endovascular coiling versus neurosurgical clipping for people with aneurysmal subarachnoid haemorrhage. Cochrane Database Syst Rev. 2018; 8: CD003085, doi: 10.1002/14651858.CD003085.pub3, indexed in Pubmed: 30110521.

4. Molyneux AJ, Kerr RSC, Yu LM, et al. International Subarachnoid Aneurysm Trial (ISAT) Collaborative Group. International subarachnoid aneurysm trial (ISAT) of neurosurgical clipping versus endovascular coiling in 2143 patients with ruptured intracranial aneurysms: a randomised comparison of effects on survival, dependency, seizures, rebleeding, subgroups, and aneurysm occlusion. Lancet. 2005; 366(9488): 809-817, doi: 10.1016/S0140-6736(05)67214-5, indexed in Pubmed: 16139655.

5. Nehls DG, Flom RA, Carter LP, et al. Multiple intracranial aneurysms: determining the site of rupture. J Neurosurg. 1985; 63(3): 342-348, doi: 10.3171/jns.1985.63.3.0342, indexed in Pubmed: 4020459.

6. Beck J, Rohde S, Berkefeld J, et al. Size and location of ruptured and unruptured intracranial aneurysms measured by 3-dimensional rotational angiography. Surg Neurol. 2006; 65(1): 18-25; discussion 25, doi: 10.1016/j.surneu.2005.05.019, indexed in Pubmed: 16378842.

7. Nagahata S, Nagahata M, Obara M, et al. Wall Enhancement of the Intracranial Aneurysms Revealed by Magnetic Resonance Vessel Wall Imaging Using Three-Dimensional Turbo Spin-Echo Sequence with Motion-Sensitized Driven-Equilibrium: A Sign of Ruptured Aneurysm? Clin Neuroradiol. 2016; 26(3): 277-283, doi: 10.1007/s00062-0140353-z, indexed in Pubmed: 25332151.

8. Kondo R, Yamaki T, Mouri W, et al. [Magnetic resonance vessel wall imaging reveals rupture site in subarachnoid hemorrhage with multiple cerebral aneurysms]. No Shinkei Geka. 2014; 42(12): 1147-1150, doi: 10.11477/mf.1436200050, indexed in Pubmed: 25433063.

9. Matouk CC, Mandell DM, Günel M, et al. Vessel wall magnetic resonance imaging identifies the site of rupture in patients with multiple 
intracranial aneurysms: proof of principle. Neurosurgery. 2013; 72(3): 492-6; discussion 496, doi: 10.1227/NEU.0b013e31827d1012, indexed in Pubmed: 23151622.

10. Karttunen Al, Jartti PH, Ukkola VA, et al. Value of the quantity and distribution of subarachnoid haemorrhage on CT in the localization of a ruptured cerebral aneurysm. Acta Neurochir (Wien). 2003; 145(8): 655-61; discussion 661, doi: 10.1007/s00701-003-0080-8, indexed in Pubmed: 14520544.

11. Latchaw RE, Silva P, Falcone SF. The role of CT following aneurysmal rupture. Neuroimaging Clin N Am. 1997; 7(4): 693-708, indexed in Pubmed: 9336494.

12. Hijdra A, Brouwers PJ, Vermeulen M, et al. Grading the amount of blood on computed tomograms after subarachnoid hemorrhage. Stroke. 1990; 21(8): 1156-1161, doi: 10.1161/01.str.21.8.1156, indexed in Pubmed: 2389295.
13. Takeuchi S, Takasato $\mathrm{Y}$, Masaoka $\mathrm{H}$, et al. [Unusual distribution of blood in a ruptured aneurysm--case report]. Brain Nerve. 2009; 61(12): 1425-1428, indexed in Pubmed: 20034310.

14. Lee KC, Joo JY, Lee KS. False localization of rupture by computed tomography in bilateral internal carotid artery aneurysms. Surgical Neurology. 1996; 45(5): 435-441, doi: 10.1016/0090-3019(95)00409-2.

15. Jing L, Fan J, Wang Y, et al. Morphologic and Hemodynamic Analysis in the Patients with Multiple Intracranial Aneurysms: Ruptured versus Unruptured. PLoS One. 2015; 10(7): e0132494, doi: 10.1371/journal. pone.0132494, indexed in Pubmed: 26147995.

16. Zhang $Y$, Yang $X$, Wang $Y$, et al. Influence of morphology and hemodynamic factors on rupture of multiple intracranial aneurysms: matched-pairs of ruptured-unruptured aneurysms located unilaterally on the anterior circulation. BMC Neurol. 2014; 14: 253, doi: 10.1186/s12883014-0253-5, indexed in Pubmed: 25551809. 